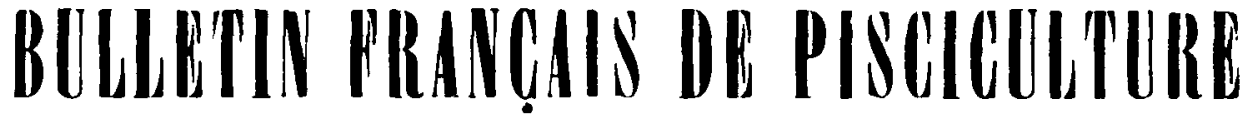

\section{ACTION DES FELILLES DE PEUPLIER DANS IE PETITS bassins DE PISCICUlture}

\author{
Par A. WURTZ \\ Chef de Travaux des Eaux et Forêts \\ chargé da la Station d'Hydrobiologie aipliquée du Paraclet.
}

L'action des feuilles d'arbres tombant dans les étangs de pisciculture ou dans les eaux courantes a déjà préoccupé un certain nombre d'auteurs. M. Huet (1950) a signalé la nocivité des boisements d'épicéas pour certains cours d'eau des Ardennes belges, et a même parlé de la stérilisation plus ou moins complète de ces cours d'eau traversant des forêts de résineux. E. Leclerc et F. Devlaminck (1950) ont montré par des expériences très précises la toxicité des essences résineuses pour les poissons : avec de l'eau calcaire dans laquelle ils ont fait tremper pendant 17 heures des aiguilles d'épicéa (10 grammes d'aiguilles pour 1 litre d'eau), ils ont observé la mort des Vairons en expérience en 10 heures; les aiguilles de pin silvestre ont tué dans les mêmes conditions les Vairons en 2 h. 30 , et le record a été battu par les feuilles de mélèze qui ont provoqué la mort en 2 h. 15. Il est donc démontré que les résines et composés terpéniques, qui se trouvent dans les aiguilles de résineux, sont nettement toxiques pour les poissons.

Pour les feuilles d'arbres autres que les résineux, les observations sont moins complètes, et en tout cas moins sévères. On n'a pas encore signalé de mortalité de Poissons due aux arbres à feuilles caduques. Cependant, en 1945, M. LefÈvre a laissé entendre que la présence massive dans les étangs de feuilles d'arbres (chêne et platane, en particulier) ou d'eau ayant lavé les sols forestiers, semble entraver la fraye de la Carpe, et attribue cette action stérilisante aux tanins des feuilles.

A l'occasion d'une question posée par une Fédération départementale de pêche au Service des Recherches Piscicoles, à savoir la nocivité des feuilles de peuplier tombant en abondance dans les étangs de pisciculture, il a été procédé à la Station d'Hydrobiologie appliquée du Paraclet à des expériences comparatives en deux bassins. Je me propose de relater ici d'une manière succincte le résultat de ces expériences qui ont été pour- 
suivies pendant dix-huit mois, de Mai 1953 à Octobre 1954. Bien que les chiffres des dosages chimiques soient assez peu concluants, en tout cas difficiles à interpréter, nous verrons que l'influence des feuilles de peuplier s'est nettement fait sentir en ce qui concerne la productivité piscicole.

Les deux bassins servant aux expériences étaient en tous points identiques; même surface : $504 \mathrm{~m}^{2}$ chacun, profonds de $50 \mathrm{~cm}$. environ à l'entrée et de 1 mètre environ à la pêcherie, alimentés par de l'eau calcaire provenant de la rivière "la Noye" (degré hydrotimétrique : 22 à 230, alcalinité à l'hélianthine exprimée arbitrairement en $\mathrm{CaO}: 120 \mathrm{mg}$. par litre). L'eau est restée stagnante en principe pendant toute la durée de l'expérience; en raison de légères fuites par les digues ou la pêcherie, il était tout au plus nécessaire de rajouter de l'eau une fois par mois, pour maintenir le niveau normal.

Le bassin appelé B. 5 a reçu les feuilles de peuplier; le bassin B. 6 a servi de témoin. La même quantité de Poissons-géniteurs ont été introduits dans les deux bassins au moment de l'empoissonnement en Avril 1953, soit respectivement 25 Rotengles et 10 Tanches. Puis, à plusieurs reprises le bassin $B .5$ a reçu une assez forte quantité de feuilles de peuplier : d'abord en Mai-Juin 1953 des feuilles mortes provenant de l'automne précédent, et d'Août à Octobre des feuilles vertes cueillies ou fraîchement tombées ; ensuite dans le courant de l'année 1954, une partie de feuilles fraîches et une partie de feuilles mortes, le tout correspondant à la valeur d'une quarantaine de sacs, ce qui représente, d'après mes estimations (les feuilles mortes ou fraîches étant plus ou moins tassées dans les sacs) un poids total d'environ $300 \mathrm{kgs}$ de feuilles, réparties uniformément sur le fond $\mathrm{du}$ bassin. On voit que la dose de feuilles a été volontairement un peu forcée, et se trouve être plus forte qu'une accumulation normale dans la nature.

\section{Commentaires SUR les DOSAGes ChIMIQUes,}

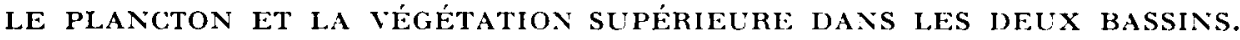

Avant de passer en revue les variations des différents facteurs chimiques et biologiques dans les deux bassins, il convient de signaler que dès le début de l'expérience, il s'est développé sur le fond du bassin B. 6 une abondante végétation de Chara (Algues vertes supérieures) alors que le bassin B. 5, qui a reçu des feuilles de peuplier, en est resté dépourvu.

On ne peut pas affirmer que l'introduction des feuilles de peuplier a été responsable de l'absence de Chara dans le bassin B. 5, car le plancton et les végétaux supérieurs sont restés à peu près identiques qualitativement dans les deux bassins, mais le développement massif de Chara dans le bassin-témoin a entraîné un certain nombre de modifications dans la composition chimique de l'eau, notamment par suite de l'assimilation chlorophyllienne : augmentation de la teneur en oxygène dissous, augmentation $\mathrm{du} p \mathrm{H}$ et diminution du degré hydrotimétrique, trois phénomènes qui sont liés et qui s'expliquent aisément. 
Plancton. - Deux mois après le début de l'expérience, le zooplancton (Cladocères : Bosmines, Daphnies ; Copépodes ; Rotifères) était beaucoup plus abondant quantitativement dans le bassin aux feuilles de peuplier, malgrè une importante différence de la teneur en oxygène dissous. Les feuilles de peuplier semblaient avoir une action favorisante, du moins temporaire. En réalité, les répercussions soit des feuilles de peuplier, soit des Chara, sur le plancton ont été à peu près inexistantes. Le phytoplancton a été identique dans les deux bassins, identique d'ailleurs aussi d'une manière générale à celui des autres étangs de la Station : petites Diatomées ubiquistes, Chrysophycées (Dinobryon), quelques Chlorococcales, petites Desmidiées ubiquistes, rares Péridiniens, au printemps (Mai-Juin) à la surface de l'eau une fleur d'eau d'Euglènes, suivie en Juillet-Août d'une fleur d'eau de Microcystis (Cyanophycées). Ajoutons à cela des touffes d'algues filamenteuses (Conjuguées et Cladophora) recouvrant pendant la saison chaude une partie de la surface des bassins, avec une abondance un peu plus grande dans le témoin.

On comprend qu'en raison du voisinage des deux bassins et de l'identité de leur plancton pendant toute la durée de l'expérience, j'aie été persuadé que l'on n'observerait pas de différence sensible dans la productivité piscicole et qu'en fin de compte les feuilles de peuplier n'auraient aucune influence.

Plantes supérieures. - Cette impression était encore confirmée par l'identité des plantes aquatiques supérieures poussant dans les deux bassins : végétation submergée formée de touffes isolées de Renoncules (Ranunculus divaricatus), de Myriophylles (Myriophyllum spicatum), quelques feuilles flottantes de Potamots (Potamogeton natans), sur les bords, quelques Roseaux (Sparganium ramosum), et au milieu du B.5, un petit îlot de Scirpus lacustris.

Le tableau 1 donne le résumé des dosages physico-chimiques effectués pendant les dix-huit mois qu'a duré l'expérience.

Matières organiques. - Tout en sachant que les chiffres relatifs aux matières organiques en solution dans l'eau (exprimés en milligrammes de permanganate de potassium consommé par litre d'eau, à chaud, en milieu alcalin) n'ont pas une valeur absolue, je fondais quelque espoir sur ces mesures, pensant que l'introduction des feuilles de peuplier se traduirait par une augmentation de la teneur en matières organiques dissoutes. Il n'en a rien été. Du moins les différences ont-elles été peu sensibles. Sur 12 dosages, 4 fois les chiffres du bassin B.5 ont été inférieurs à ceux du témoin. A signaler les deux chiffres élevés du 21-1-54 (respectivement 28 et $24 \mathrm{mg}$.) que l'on peut expliquer par l'arrivée d'eau charriant un peu de vase provenant de la rivière, cette alimentation mensuelle étant rendue nécessaire, comme j'ai déjà eu l'occasion de le dire, pour maintenir le niveau constant dans les bassins.

Dans la plupart des cas, les chiffres de la teneur en matières organiques du bassin aux feuilles de peuplier ont été légèrement supérieurs à ceux du témoin, sans toutefois que cette différence ait été assez sensible 
pour permettre de conclure à une action nette des feuilles. Des chiffres de l'ordre de 4 à 7 milligrammes de matières organiques sont faibles et fort peu concluants. A deux reprises les chiffres (respectivement 2,2 et $3,4 \mathrm{mg}$. le 27-8-53, puis 3,3 et $3,1 \mathrm{mg}$. le 23-2-54) ont été très bas, et ont mème caractérisé une eau particulièrement pure, assez surprenante pour de petits étangs peu profonds.

Oxygène dissous, $\mathrm{pH}$ et degré hydrotimétrique. - Comme on pouvait s'y attendre dans de petites pièces d'eau et comme cela a déjà été montré dans un travail antérieur (A. Wurtz et J. WLRTZ-ARLET, 1948), la teneur en oxygène dissous et le $p H$ subissent de fortes variations; on a ajouté dans l'expérience relatée ici les variations du degré hydrotimétrique. En étudiant les variations journalières de la teneur en oxygène dissous dans les étangs du Paraclet, nous savions déjà que celle-ci pouvait osciller entre les limites extrêmes de $63 \%$ et $184 \%$ de la saturation, et que le $p H$ pouvait varier en un jour dans un même étang de 7,1 à 8,2 .

Ici, les variations extrèmes sont comprises pour l'oxygène entre $45 \%$ et $226 \%$ de la saturation, et pour le $p H$ entre 7,1 et 9 . Le chiffre de $20,6 \mathrm{mg}$. d'oxygène dissous, remarquablement élevé (correspondant à $226 \%$ de la saturation), a été atteint le 10-7-53 et est dù à l'intense assimilation chlorophyllienne des Chara recouvrant le fond du bassin-témoin comme un tapis. Au début encore, on a pu croire à une action nette des feuilles de peuplier, car à ce chiffre élevé de $20,6 \mathrm{mg}$., s'opposaient les $4,15 \mathrm{mg}$. d'oxygène dissous du B.5 (correspondant à $45 \%$ de la saturation seulement); il semblait assez clair que les feuilles de peuplier provoquaient des fermentations et une absorption d'oxygène. Mais par la suite les différences se sont estompées et le bassin B.5 a également largement dépassé la saturation, effaçant ainsi l'influence possible des feuilles de peuplier.

Le bassin témoin, envahi de Chara, a eu presque continuellement des teneurs en oxygène très élevées $(127,145), 162,192$, 200 et enfin $226 \%$ de la saturation), sauf une première fois, le $12-5-1954$ où les $7,8 \mathrm{mg}$. ( $84 \%$ de la saturation) correspondent à une décomposition temporaire d'algues filamenteuses et de plancton, et les deux derniers chiffres, de juillet à septembre 1954 (5 et $4,3 \mathrm{mg}$. d'oxygène qui ne représentent plus que $56 \%$ et $47 \%$ de la saturation) qui sont dues à la mort et à la décomposition, effectivement visibles, du tapis de Chara. Le bassin B.5, malgré les feuilles, a atteint $17,8 \mathrm{mg}$. d'oxygène par litre $(143 \%$ de la saturation le 21-1-1954 et, sur 12 dosages, 9 se sont maintenus au-dessus de la saturation, entre $113 \%$ et 143 à $145 \%$ de la saturation); à la fin de l'expérience seulement (les phénomènes de décomposition ont entraîné un abaissement considérable de la teneur en oxygène $(5,7 \mathrm{mg}$., soit $54 \%$ de la saturation) sans que l'on puisse différencier d'ailleurs si les phénomènes en question sont dus aux feuilles mortes ou aux plantes supérieures.

En ce qui concerne le $p I$, il est normal que l'on observe des variations en rapport avec l'assimilation chlorophyllienne, sans qu'il soit nécessaire d'insister plus longuement sur les chiffres que l'on peut lire sur le tableau 1. 


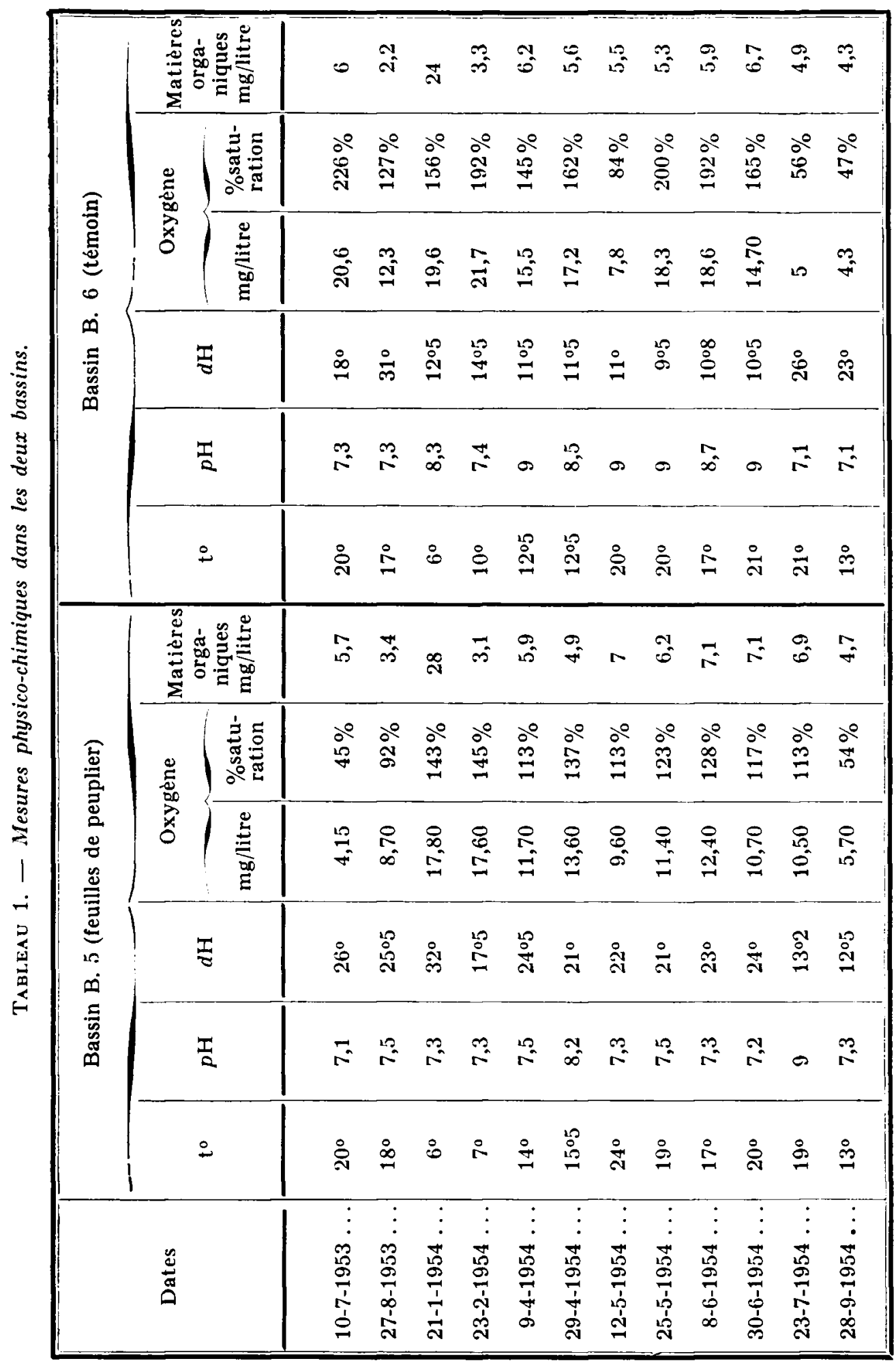


On sait que dans les pièces d'eau peu tamponnées, les variations peuvent être assez fortes, uniquement par l'action des phénomènes biologiques se répercutant sur l'équilibre carbonates-bicarbonates-gaz carbonique. Ici, pendant la période d'intense développement des Chara dans le bassintémoin, le $p H$ varie entre 7,3 et 9 ; au moment de la mort et de la décomposition des Chara, il tombe à 7,1 , ce qui montre que lorsque l'assimilation chlorophyllienne s'arrête, il y a une tendance vers l'acidification, sans que toutefois le $p \mathrm{H}$ puisse devenir inférieur à 7 . Dans le bassin B.5, à 9 reprises le $p H$ était légèrement inférieur à celui du bassin-témoin, ce qui reviendrait à dire que les feuilles de peuplier avaient une action acidifiante; en réalité, c'est l'absence de Chara et l'assimilation chlorophyllienne moins intense dans le B.5 qui a déterminé le $p H$ plus bas. II a suffi d'un bon développement de Myriophylles et d'une journée ensoleillée pour que, dans le bassin aux feuilles de peuplier, le $p \mathrm{H}$ monte aussi à 9 .

Pour compliquer le tableau et montrer que rien n'est simple en hydrobiologie, ajoutons encore quelques mots sur le degré hydrotimétrique, dont les variations ont été extrêmement intéressantes. La mesure du degré hydrotimétrique n'étant pas d'une précision rigoureuse, nous admettrons que, dans les étangs, des valeurs comprises entre $22^{\circ}$ et $26^{\circ}$ sont normales et nous nous interrogerons sur la signification des valeurs qui se trouvent au-dessus et au-dessous de cette moyenne assez large.

Éliminons d'abord les deux chiffres aberrants ( $31^{\circ}$ dans le $\mathrm{B} .6$ le 27-8-1953), et $32^{\circ}$ dans le B.5 le 21-1-1954) qui ne peuvent s'expliquer que par une arrivée intempestive de sels alcalino-terreux à la suite de l'alimentation intermittente des bassins par la rivière, ou une mobilisation (solubilisation) de ces mêmes sels à partir du fond calcaire et tourbeux des bassins. Cela nous permettra de constater que les feuilles de Feuplier n'ont pas eu d'influence sur le degré hydrotimétrique car celui-ci s'est maintenu, sauf pour les deux derniers chiffres, près de la moyenne. 1) ans le bassin B.6 par contre, les Chara ont encore amené des perturbations assez importantes : pendant toute la durée de leur développement intense et de l'assimilation chlorophyllienne (même en hiver, dans l'eau à $6^{\circ}$ ), le degré hydrotimétrique est tombé à $12^{\circ} 5,11^{\circ}, 10^{\circ} 5$ et même $9^{\circ} 5$, ce qui correspond à un important " départ " des sels alcalino-terreux. Ce phénomène est bien connu; c'est la " précipitation biogénique " du carbonate de calcium : par suite de l'assimilation chlorophyllienne et de l'absorption de $\mathrm{CO}^{2}$ par les plantes, les bicarbonates solubles précipitent sous forme de carbonate de calcium insoluble, recouvrant les touffes de Chara ou les feuilles de plantes submergées d'une pellicule ou même d'une croùte blanche de "calcaire " qui n'est plus dosé lorsque l'on mesure le degré hydrotimétrique. Le carbonate de calcium ainsi précipité n'est d'ailleurs pas définitivement hors cycle car il peut être repris dans le cycle et solubilisé par des acides organiques (provenant des décompositions dans la vase) ou par du gaz carbonique agressif.

L'illustration de ce fait est fournie par les deux derniers chiffres du B.6, correspondant à la mort et la décomposition des Chara : l'assimilation chlorophyllienne cessant, le degré hydrotimétrique retrouve 
sa valeur normale. Enfin les deux derniers chiffres du bassin B.5 montrent que, malgré les feuilles de peuplier, la présence d'une certaine quantité de Myriophylles provoque à son tour une précipitation biogénique de carbonate de calcium.

Ces commentaires m'ont éloigné du problème des feuilles de peuplier et peuvent sembler un peu longs parce qu'il est difficile d'exposer successivement des phénomènes qui ont lieu simultanément et qui dépendent même assez étroitement les uns des autres. Ils auront eu l'intérêt de montrer deux choses sur lesquelles il était bon d'insister : d'abord, que dans des petits bassins de pisciculture, les végétaux submergés jouent un rôle important par leur assimilation chlorophyllienne, laquelle agit sur la teneur en oxygène dissous, le $p \mathrm{H}$, le degré hydrotimétrique. Ensuite que les feuilles de peuplier, introduites en abondance dans l'un des bassins, n'ont pas eu de répercussions appréciables sur la composition chimique de l'eau libre, sur le plancton et la végétation en général. Rien ne laissait prévoir que les deux bassins auraient une productivité piscicole différente.

Il a fallu attendre la pêche et la vidange totale des deux bassins en octobre 1954 pour nous rendre compte et comprendre enfin comment les feuilles de peuplier avaient agi.

\section{RÉsultats de la PÊche DANS LES DEUX BASSins.}

Dans les chiffres qui vont être donnés, il n'a pas été tenu compte des géniteurs dont l'accroissement en poids a été très faible. Comme il ne s'agissait pas d'étudier des problèmes de production quantitative de plancton ou de benthos, et que l'essentiel était de voir comment se reproduiraient les Poissons et quelle serait la production d'alevins, la nourriture et l'augmentation en poids des géniteurs ont pu être considérés comme négligeables. Les alevins par contre ont été comptés, mesurés avec beaucoup de soin. Le tableau 2 résume les chiffres relatifs à la production d'alevins dans les deux bassins.

Le bassin B.6 (témoin, sans feuilles de peuplier), malgré la présence au moment de la vidange d'une grande quantité de Chara plus ou moins en décomposition sur le fond, a produit 27,2 kilogrammes de Poissons (15 kilogrammes d'alevins de Tanches, et 12,2 kilogrammes d'alevins de Gardons), soit 170 Tanches de $14 / 16 \mathrm{~cm} ., 600$ Tanches de $10 / 12 \mathrm{~cm}$., 3.300 Gardons de $6 / 9 \mathrm{~cm}$. en moyenne, et quelques rares Gardons de $12 / 13 \mathrm{~cm}$. Pour donner une productivité à l'hectare, il suffit de multiplier tous ces chiffres par 20 , ce qui nous donne une production assez élevée de 544 kilogrammes d'alevins, à l'hectare, extrapolation permise pour nous donner un chiffre relatif, mais qui se révèle fausse en pratique, car on sait que la productivité d'alevins est toujours plus forte, à surface égale, dans de nombreux petits bassins que dans un seul et grand étang.

Le bassin B.5, avec les feuilles de peuplier, renfermait à la vidange une énorme quantité de vase putride, d'odeur nauséabonde et n'a produit 
que 10 kilogrammes de Poissons (5 kilogrammes de Tanches et 5 kilogrammes d'alevins de Gardons), soit 275 Tanches de $12 / 14 \mathrm{~cm}$, et 1.250 Gardons de $6 / 8 \mathrm{~cm}$. en moyenne, avec quelques rares Gardons de $4 \mathrm{~cm}$., et, toujours en extrapolant, un poids total de 200 kilogrammes d'alevins à l'hectare. La production du bassin B.5 a donc été plus de deux fois et demie inférieure à celle du témoin; les alevins ont été en moyenne moins gros et beaucoup moins nombreux (1).

Les feuilles de peuplier en décomposition ont provoqué la formation d'une abondante quantité de vase putride sur le fond du bassin B.5; cette vase a éliminé la faune benthique nutritive, intéressante pour les Poissons, a changé les conditions de reproduction et diminué d'une manière appréciable la productivité.

Cette constatation éclaire sous un jour nouveau les problèmes de la biologie et de la production d'alevins ou de Poissons en général dans les étangs. Il y a d'une part les phénomènes qui se passent en eau libre et qui intéressent les êtres planctoniques, les plantes submergées et les Poissons planctophages ou herbivores liés à ce mode de nutrition, phénomènes qui dépendent de la vie autotrophe des plantes, de la lumière solaire et des sels minéraux dissous. Il y a d'autre part ce qui se passe au niveau de la vase (chimie complexe des décompositions, putréfactions, minéralisations par chimiosynthèse, réductions et hétérotrophies), phénomènes qui intéressent les êtres benthiques du fond et en dernier ressort les Poissons qui se nourrissent de ces êtres benthiques ou qui ont besoin du fond pour se reproduire.

Les rapports entre ces deux milieux et ces deux groupes de phénomènes sont complexes et, dans l'état actuel de nos connaissances, à peu près impossibles à prévoir. Les échanges entre la vase et l'eau sont en tous les cas lents ; même dans les petits étangs la vase est un réservoir qui ne lâche que lentement les substances qu'elle fabrique ou transforme. Et lorsque la formation de vase est normale, il se peut fort bien qu'il n'y ait aucune répercussion sur la productivité piscicole.

Si, par contre, il y a une accumulation anormale de vase dans l'eau, due à un apport trop important de matières organiques (végétaux en décomposition, feuilles d'arbres dans le cas présent, matières putrescibles provenant d'une usine lointaine), il peut y avoir une véritable pollution, avec élimination de la faune du fond intéressante et abaissement de la productivité piscicole.

C'est ce qui s'est passé avec les feuilles de peuplier. Pendant toute la durée de l'expérience où le bassin B.5 est resté en eau, il s'est accumulé une couche de plus en plus épaisse de feuilles mortes en décomposition,

(1) En ce qui concerne l’àge exact de ces alevins, précisons que la majorité des alevins de Gardons de $4 / 9 \mathrm{~cm}$. avaient un été et provenaient de la ponte de 1954. Quelques rares alevins de plus de $12 \mathrm{~cm}$. ont été reconnus avoir deux étés. On a tout lieu de croire que la fraye de 1953 s'est faite dans de mauvaises conditions ou que les alevins correspondants ont disparu, Quant aux Tanches, dont la scalimétrie est difficile, il semble que les rares alevins de $6 / 9 \mathrm{~cm}$. soient de première année (1954), et la majorité des $11 / 14 \mathrm{~cm}$. et $14 / 16 \mathrm{~cm}$. de deuxième année (1953); pour les Tanches c'est la fraye de 1953 qui a été la meilleure. 


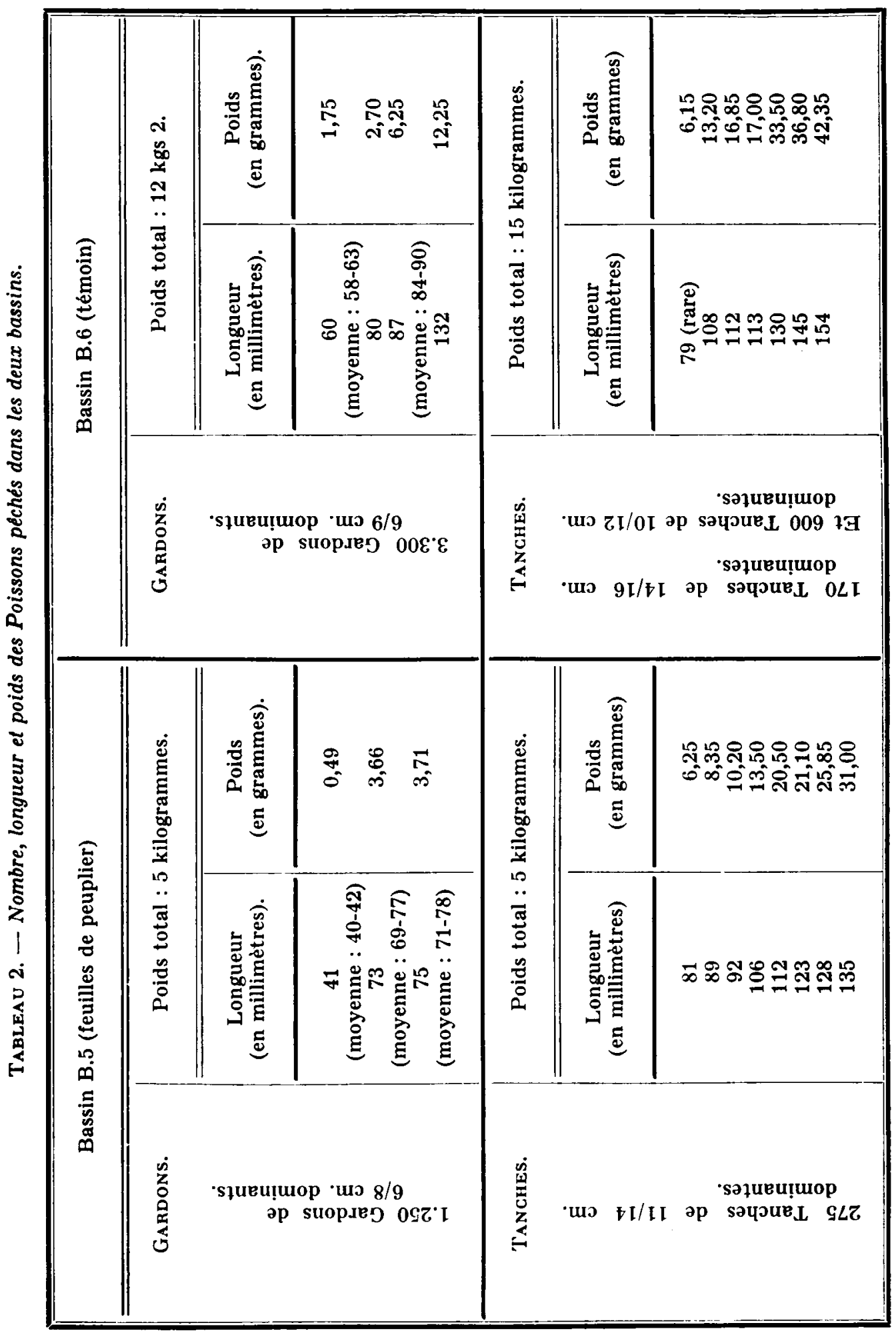


formant une vase putride extrêmement pauvre en animaux benthiques (par exemple Mollusques, Vers, larves d'Insectes, etc.). On a suffisamment insisté dans les commentaires précédents sur le fait que les feuilles de peuplier n'avaient eu aucune répercussion chimique ou biologique décelable dans l'eau libre du bassin B.5. Il est donc certain que la vase putride n'a pas làché de substances fermentescibles, ni de gaz; les feuilles n'ont pas produit de substances toxiques et n'ont pas empoisonné les Poissons. Et pourtant il a suffi de vider le bassin pour que la vase dégage une odeur nauséabonde d'hydrogène sulfuré et d'ammoniaque.

Les produits de décomposition, de putréfaction des feuilles se trouvaient dans la vase; celle-ci était effectivement putride, mais en raison de la lenteur des échanges dont il vient d'etre question, ces produits n'ont pas diffusé dans l'eau libre, et celle-ci a donné l'impression d'une eau d'étang restée à peu près pure.

En réalité nous nous trouvons en présence d'une pollution organique typique par envasement : il y a formation d'une trop grande quantité de vase d'origine exogène, puis début de fermentation, transformation en vase putride et anaérobie, élimination de la faune benthique et abaissement de la capacité biogénique de la pièce d'eau considérée.

La nocivité de l'excès de feuilles de peuplier étant ainsi établie, en ayant volontairement, comme je l'ai dit au début, forcé la dose, la question est de savoir si dans la nature pareil phénomène peut se produire, et si une accumulation normale, naturelle, de feuilles de peuplier dans un étang peut être nocive. Autrement dit un étang, entouré de peupliers, dans lequel tombent des feuilles de peuplier, sera-t-il moins bon au point de vue pisciculture qu'un autre étang dans lequel il n'en tombe pas?

Deux observations faites au Paraclet permettent de répondre à cette question. Il y a d'abord le cas d'un de nos étangs d'alevinage, d'une surface de 60 ares, appelé $E$. 5, dans lequel nous produisions normalement des alevins de Gardons, ou de Carpes, de Tanches, et le cas échéant de Black-bass. C'est le seul de nos étangs bordé de peupliers, dans lequel tombe chaque année une quantité appréciable de feuilles. Il y a trois ans, et les années antérieures, cet étang avait encore une productivité assez bonne. Depuis cette époque, il s'est considérablement envasé ; depuis deux ans, les poissons ne s'y reproduisent plus et sa productivité en alevins est réduite à zéro, alors que les autres étangs du Paraclet non entourés de peupliers gardent leur bonne productivité moyenne. Cet étang ne pourra être remis en état qu'en draguant plus de $500 \mathrm{~m}^{3}$ de vase. Nous avons là un exemple typique de l'accélération de l'envasement d'un étang par les feuilles de peuplier, et de l'influence nocive de celles-ci, empêchant par leur accumulation la reproduction des Poissons, réduisant la capacité biogénique dans des proportions considérables, et transformant un étang qui était bon en un étang absolument impropre à la pisciculture.

L'autre cas s'est produit dans la source d'eau pure alimentant la salmoniculture du Paraclet. En effet, nos sources, après leur captage, traversent sur une trentaine de mètres une série de bassins à ciel ouvert 
avant d'alimenter soit les bassins cimentés où sont maintenus les géniteurs-Truites, soit par une canalisation cimentée, la salmoniculture proprement dite. Il va sans dire que cette eau a toujours été pure et convient parfaitement à l'élevage des Truites. Jusqu'en automne 1954 du moins ! En effet, sur la trentaine de mètres de bassins à ciel ouvert, sorte de petite rivière fort agréable, renfermant quelques Truites, des Chabots, des Épinoches et une végétation supérieure typique de source d'eau pure, il tombe des feuilles de peuplier et les phénomènes de décomposition dont les feuilles ont été le siège au fond de l'eau ont été jusqu'à inquiéter nos Truites. A partir de la deuxième quinzaine d'Octobre 1954, les Truites se trouvant à plus de 30 mètres de l'endroit de la chute des feuilles, ont commencé à venir piper en surface et donner des signes manifestes d'asphyxie. Un dosage d'oxygène nous a donné $8 \mathrm{mg} / \mathrm{litre}$, ce qui correspond pour la température de $11^{\circ}$ à $70 \%$ de la saturation ; le B.O.D. de cette eau était de $1,5 \mathrm{mg} /$ litre, ce qui n'est peut-être pas très élevé, mais indique néanmoins des phénomènes anormaux pour une eau pure. Remontant vers la source, les chiffres nous donnèrent respectivement $50 \%$ de la saturation à l'entrée du bassin à Truites, et $76 \%$ de la saturation (mais avec un B.O.D. de $2,1 \mathrm{mg} / \mathrm{litre}$ ) à l'endroit où tombent le plus de feuilles (chiffres du 7-11-1954).

L'indice de putrescibilité de l'eau est resté négatif ; il n'y avait pas de trace d'hydrogène sulfuré dans l'eau. Mais instruit par l'expérience du bassin B. 5 qui venait d'être vidé et dans lequel on avait constaté après coup l'énorme accumulation de vase putride, j'ai prélevé, avec un appareil spécial, de la vase au fond du bassin "d'eau pure " qui présentait la plus faible teneur en oxygène. La vase était noire, dégageait une intense odeur d'hydrogène sulfuré, et il y avait des bactéries sulfuraires. Les feuilles de peuplier sont donc arrivées à polluer l'eau la plus pure, et à incommoder des Truites. Nous avons pris les mesures radicales qui s'imposaient : couper les peupliers bordant les bassins et sortir le plus possible de vase putride avec des dragues maniées péniblement à la main.

Dans les cours d'eau bordés de peupliers, pareils phénomènes peuvent également se produire, et il est difficile de mettre en évidence les pollutions insidieuses, par envasement " naturel ", occasionnées par l'accumulation de feuilles au fond des rivières et formation lente de vase putride. L'analyse chimique de l'eau coulant tranquillement sur cette vase (comme j'ai eu l'occasion de le montrer à plusieurs reprises au cours de cet article) ne donne pratiquement pas d'indications valables; il faut des études minutieuses de la vase, des bactéries qui la peuplent, des fermentations dont elle est le siège ; il faut tenir compte de l'abaissement de la capacité biogénique des rivières par raréfaction de la faune benthique, de l'impossibilité des poissons à se reproduire et à trouver des bonnes frayères.

Je conclus donc que, quelles que soient les eaux, étangs ou cours d'eau, la chute des feuilles de peuplier provoque au bout d'un temps plus ou moins long l'abaissement de la valeur piscicole de ces eaux, par le simple mécanisme d'une pollution organique par envasement. 


\section{RÉSUMÉ.}

Il a été procédé à des essais dans des bassins de pisciculture de mêmes dimensions $\left(504 \mathrm{~m}^{2}\right.$ ) en vue d'établir la nocivité des feuilles de peuplier. L'influence de celles-ci ne s'est guère fait sentir par les dosages chimiques effectués dans l'eau.

Par contre, les feuilles en décomposition ont provoqué une abordante quantité de vase putride sur le fond $\mathrm{du}$ bassin en expérience et le rendement en alevins de poissons a été fortement diminué dans le bassin avec feuilles de peuplier : 10 kilogrammes seulement par rapport aux $27 \mathrm{~kg} .200 \mathrm{du}$ bassin-témoin. On peut conclure que les feuilles de peuplier, tombant en abondance dans les étangs sont nettement nocives, tant pour la faune benthique du fond, qui est éliminée par suite de la formation de vase putride, que pour la productivité piscicole.

Des observations complémentaires permettent de penser que les étangs et même les cours d'eau dans lesquels tombent des feuilles de peuplier sont moins bons pour l'alevinage et la productivité piscicole que ceux dans lesquels il n'en tombe pas. 